\title{
Evolving Paradigms in the Management and Outcomes of Sarcomatoid Renal Cell Carcinoma in the Era of Immune Checkpoint Inhibitors
}

\author{
Ragia Alya, g, Amandeep S. Aujla ${ }^{\mathrm{b}}$, Sachin Guptac, Ruby Gupta ${ }^{\mathrm{d}}$, \\ Sorab Gupta ${ }^{\mathrm{e}}$, Sheila Kalathil ${ }^{\mathrm{f}}$
}

\begin{abstract}
Renal cell carcinoma (RCC) is a common cancer that affects a significant number of patients every year around the world. The presence of sarcomatoid features in these tumors is considered a poor prognostic feature. Patients with RCC with sarcomatoid features had significantly worse outcomes when treated with sunitinib, the previous first-line standard of care therapy when compared to patients without such features. Multiple immune checkpoint inhibitors have recently been approved for the treatment of RCC. In this article, we review the literature available on the outcomes of patients with sarcomatoid RCC treated with immune checkpoint inhibitors.
\end{abstract}

Keywords: Sarcomatoid; Renal cell carcinoma; Immune checkpoint inhibitors; Sunitinib

\section{Introduction}

The American Cancer Society estimates about 73,750 new cases of kidney and renal pelvis cancers, with about 14,830 deaths in the United States of America in 2020 [1]. Globally, these

Manuscript submitted August 8, 2020, accepted August 29, 2020

Published online October 15, 2020

${ }^{a}$ Department of Internal Medicine, Danbury Hospital, 24 Hospital Ave., Danbury, CT 06810, USA

${ }^{b}$ Department of Hematology and Medical Oncology, Hartford Healthcare Cancer Institute, 326 Washington Street, Norwich, CT 06360, USA

'Department of Internal Medicine, Tower Health Reading Hospital, 420 S 5th Ave., West Reading Hospital, West Reading, PA 19611, USA

dDepartment of Hematology and Medical Oncology, William Beaumont Hospital, Royal Oak, MI 48073, USA

eDepartment of Hematology and Medical Oncology, Bronx Care Hospital, 1650 Grand Concourse, Bronx, NY 10457, USA

fDepartment of Hematology and Medical Oncology, Einstein Medical Center, 5501 Old York Road, Philadelphia, PA 19141, USA

gCorresponding Author: Ragia Aly, Department of Internal Medicine, Danbury Hospital, 24 Hospital Ave., Danbury, CT 06810, USA.

Email: ragia.aly@gmail.com

doi: https://doi.org/10.14740/wjon1325 constitute $2.2 \%(403,262)$ of all new cancer cases with almost $1.8 \%(175,098)$ deaths associated with them [2]. The median age of onset for this disease is around 64 years, affecting males almost twice as frequently as females $[1,2]$. There are different histopathological variants of renal cell carcinoma (RCC), which constitutes the majority of kidney cancers. The most common type is the clear cell renal cell carcinoma (ccRCC), constituting about $70-75 \%$ of all cases. It is followed by papillary (10-15\%), chromophobe (5\%), collecting duct renal cell carcinomas (1\%) and medullary carcinoma (1\%) [3-5]. Sarcomatoid renal cell carcinoma (sRCC), which was previously considered a separate histological variant, is now regarded as a dedifferentiated carcinoma and can be associated with any RCC subtype [6]. The main feature of sarcomatoid type is the presence of pleomorphic spindle cells on the histopathological examination, which might also include rhabdoid, leiomyomatous, or fibrous components [6,7] and American Joint Committee on Cancer (AJCC) (2017) staging has included these as histological grade 4 features. This sarcomatoid dedifferentiation can be seen in about $1-10 \%$ of RCCs and is associated with more aggressive and relatively poor clinical outcomes [8-10].

The prognosis in advanced RCC has been defined using International mRCC Database Consortium (IMDC) and Memorial Sloan Kettering Cancer Center (MSKCC) or Motzer score. These are based on quantification of number of risk factors individually associated with poor prognosis in RCC patients: Karnofsky score $<80 \%,<12$ months from diagnosis to systemic treatment, anemia, neutropenia, thrombocytosis, hypercalcemia and elevated lactate dehydrogenase. The median overall survival (OS) in advanced RCC treated with sunitinib in pre-immunotherapy era is reported as about 43.2 to 97 months, 22.5 to 33.5 months and 5.1 to 10 months in respective IMDC favorable (no risk factors), intermediate (1 - 2 risk factors) and poor risk groups (three or more risk factors) [11-14]. The prognostic impact of sarcomatoid features have also been reported in multiple retrospective studies. Kyriakopoulos et al looked at the outcomes of 2,286 patients with RCC, of which 230 patients had sRCC subtype. The average duration between the initial diagnosis and relapse was 18.8 months among sRCC, which was significantly shorter than 42.9 months reported in non-sRCC patients [9]. Similar findings were reported by Mian et al, which reported that $77 \%$ of sRCC had already metastasized at the initial presentation, and 
Table 1. Four Clinical Phase III Randomized Controlled Trials That Compared ICls to Sunitinib

\begin{tabular}{lllll}
\hline & Checkmate 214 & IMmotion 151 & KEYNOTE 426 & JAVELIN renal 101 \\
\hline Drugs compared & $\begin{array}{l}\text { Ipilimumab/nivolumab } \\
\text { vs. sunitinib }\end{array}$ & $\begin{array}{l}\text { Atezolizumab/bevacizumab } \\
\text { vs. sunitinib }\end{array}$ & $\begin{array}{l}\text { Axitinib/pembrolizumab } \\
\text { vs. sunitinib }\end{array}$ & $\begin{array}{l}\text { Axitinib/avelumab } \\
\text { vs. sunitinib }\end{array}$ \\
N & 60 vs. 52 & 68 vs. 74 & 51 vs. 54 & 47 vs. 61 \\
ORR, $\%$ & 56.7 vs. 19.2 & 49 vs. 14 & 58.8 vs. 31.5 & 46.8 vs. 21.3 \\
CR, $\%$ & 18.3 vs. 0 & 10 vs. 3 & NR & NR \\
PFS, months & 8.4 vs. 4.9 & 8.3 vs. 5.3 & 12 months OS: & 7.4 vs. 4.0 \\
OS & Median OS: 31.2 & Median OS: 21.7 vs. & $83.4 \%$ vs. $79.5 \%$ & 12 months OS: \\
& vs. 13.6 months & 15.4 months & $83 \%$ vs. $67 \%$ \\
\hline
\end{tabular}

$\mathrm{N}$ : number of subjects with sRCC; ORR: objective response rate; CR: complete response rate; PFS: progression-free survival; OS: overall survival; NR: not reported; ICl: immune checkpoint inhibitor; sRCC: sarcomatoid renal cell carcinoma.

the median OS was about 8.5 months in these patients [15]. Sarcomatoid features along with larger tumor size (T3-4) have also been significantly associated with lymph node invasion at the time of radical nephrectomy. Among patients with localized RCCs undergoing nephrectomy with no residual disease but noted to have sarcomatoid features on pathology, $77 \%$ experienced recurrence with a median OS of only 2 years [16].

Immune checkpoint inhibitors (ICIs) have recently changed the treatment landscape of RCCs. These drugs are the monoclonal antibodies which target programmed death 1 (PD1)/programmed death ligand 1 (PDL1) and cytotoxic T-lymphocyte antigen 4 (CTLA-4) pathways, which are normally upregulated to protect normal tissues from excessive inflammation by inhibiting T-cell activation but also used by cancer cells to escape T-cell mediated anti-tumor immune responses [17]. For example, CTLA-4 is upregulated on the T cells in feedback to T-cell receptor (TCR) stimulation and engagement. It attenuates the TCR signaling by competing with the costimulatory molecule CD28 for the B7 ligands on the antigen presenting cells (APCs). CTLA-4 has higher affinity for the B7 ligands compared to CD28, thus effectively attenuating T-cell activation. Thus, tumor infiltrating APCs are better able to engage T cells in presence of CTLA-4 blockade. PD1 is also expressed on the T cells upon activation and PDL1 is expressed on APCs in response to interferon-gamma (IFN- $\gamma$ ) and on the cancer cells. PD1/PDL1 engagement inhibits T-cell activation via tyrosine phosphatase (SHP2)-based de-phosphorylation of activating signaling molecules in the T cells [18-21]. Some of the factors and biomarkers that have been shown and are being studied to predict a response to ICIs include tumor PDL1 expression, tumor immunogenicity and lymphocytic infiltration, tumor mutational burden (TMB) and deficiency in DNA mismatch repair genes (dMMR). Although $\mathrm{dMMR}$ and $\mathrm{TMB} \geq 10$ mutations/megabase have been approved by FDA as indications for use of pembrolizumab in unresectable or metastatic solid tumors irrespective of tumor site, but the only marker that has the strongest evidence towards predicting the response to all the different ICIs has been the PDL1 expression. The reliability of PDL1 as a predictive biomarker has been more consistent at its higher expression levels compared to lower expression levels and thus the quest to find a better predictive biomarker still continues. SRCC tumors have been reported to elicit significantly higher PDL1, PD1 and CD8 positive lymphocyte density in comparison to non-sarcomatoid grade 4 ccRCC. Joseph et al reported higher PD1 positive tumor infiltrating lymphocytes (96\% versus 62\%) and higher PDL1 expression (54\% versus $17 \%$ ) in sRCC compared to ccRCC [2224]. These findings are exciting for sRCC which historically has poor responses to the anti-angiogenic therapies.

Multiple ICIs (PDL1 inhibitors: atezolizumab, avelumab and durvalumab; PD1 inhibitors: nivolumab, pembrolizumab and cemiplimab; CTLA-4 inhibitors: ipilimumab) have been developed and FDA approved for numerous indications [25, 26]. In advanced RCC, ICIs are currently approved as monotherapy (nivolumab), in combination (nivolumab plus ipilimumab) and along with tyrosine kinase inhibitors (TKIs) (avelumab/pembrolizumab plus axitinib) at different lines of treatment. We have reviewed the literature and current evidence on the role of ICI in the treatment of SRCC (RCC with sarcomatoid dedifferentiation), which historically has had poor outcomes with TKIs monotherapy [27].

\section{Review}

Five recent clinical trials have studied the efficacy of multiple ICIs either as a single agent, in combination with a second ICI, or in combination with a vascular endothelial growth factor (VEGF) inhibitor in the treatment of advanced RCC. Four of these trials (Table 1) were phase III randomized controlled trials that compared ICIs to sunitinib (standard of care at the time of enrollment). These trials enrolled patients with advanced $\mathrm{RCC}$ who were treatment-naive.

\section{Checkmate 214}

Checkmate 214 is a phase III randomized open-label study comparing the efficacy of nivolumab plus ipilimumab vs. sunitinib among patients with untreated advanced or metastatic RCC. Patients were treated with nivolumab plus ipilimumab intravenously every 21 days for four doses, followed by nivolumab every 14 days, or sunitinib $(50 \mathrm{mg}$ ) orally once daily for 4 weeks (6-week cycle). The objective response rate (ORR) was significantly higher ( $42 \%$ versus $27 \%$ ) in the combination arm. At 18 months, $75 \%$ of the patients were alive in nivolumab/ip- 
ilimumab combination arm compared to $60 \%$ in sunitinib arm (hazard ratio (HR) for death was $0.63, \mathrm{P}<0.001$ ) [28]. A post hoc analysis of this study for patients with sarcomatoid RCC subset identified 112 patients with sRCC, of which 60 patients were randomized to the nivolumab/ipilimumab arm and 52 to the sunitinib arm. The ORR was $56.7 \%$ in patients who received nivolumab/ipilimumab compared to only $19.2 \%$ in the sunitinib arm. The complete response rate (CR) was $18.3 \%$ in nivolumab/ ipilimumab arm and $0 \%$ in the sunitinib arm. Patients in the combination arm had significantly higher OS and median progression-free survival (PFS) [29]. The combination is currently recommended as first-line treatment for patients with clear cell $\mathrm{RCC}$ who are classified as IMDC poor or intermediate risk by the National Comprehensive Cancer Network.

\section{KEYNOTE 426}

This is a phase III randomized open-label study that compared the efficacy of pembrolizumab plus axitinib for the treatment of advanced, previously untreated RCC in comparison to sunitinib monotherapy. A total of 861 patients underwent randomization, with 432 patients assigned to the combination arm, and 429 patients assigned to the sunitinib arm. The ORR was $59.3 \%$ in the pembrolizumab-axitinib group and $35.7 \%$ in the sunitinib group with a CR of $5.8 \%$ of patients in the pembrolizumab-axitinib group and $1.9 \%$ in the sunitinib group. On subgroup analysis presented at the ASCO 2019 meeting, out of the 861 patients enrolled in the trial, 105 had RCC with sarcomatoid dedifferentiation, of which 51 patients were randomized to pembrolizumab plus axitinib arm and 54 to the sunitinib arm. Pembrolizumab plus axitinib arm was reported to have a higher OS of $58.8 \%$ compared to $31.5 \%$ in the sunitinib arm. PFS and OS were favorable with ICI-combination arm, but the difference did not meet statistical significance (PFS HR 0.54, 95\% confidence interval (CI) 0.29 - 1.00; OS HR 0.58, 95\% CI 0.21 - 1.59). This clinical trial has led to the approval of pembrolizumab plus axitinib as a first-line treatment of advanced clear cell RCC in all IMDC risk categories [30].

\section{KEYNOTE 427}

This is a phase II single-arm open-label monotherapy clinical trial on the use of pembrolizumab for locally advanced/metastatic RCC. The study has two cohorts: cohort A included patients with clear cell RCC, with a total number of 110 patients, and cohort B included patients with non-clear cell RCC with a total number of 165 patients. Cohort $A$ included 11 patients with sarcomatoid features, and these patients had an ORR of $64 \%$ with a disease control rate of $73 \%$ [31]. Cohort B included 38 patients with sarcomatoid RCC, with an analysis of this subgroup revealing an ORR of $44.7 \%$ [32].

\section{IMmotion 151}

This is a phase III open-label randomized controlled trial that compared atezolizumab $(1,200 \mathrm{mg})$ plus bevacizumab $(15 \mathrm{mg} /$ $\mathrm{kg}$ ) every 3 weeks to sunitinib (50 mg daily for 4 weeks on/2 weeks off) in patients with treatment-naive unresectable locally advanced or metastatic RCC with any component of clear cell or sarcomatoid histology. A total of 915 patients participated in the study, 454 patients were randomly assigned to the atezolizumab plus bevacizumab arm, and 461 patients were assigned to the sunitinib arm. Out of the 915 patients enrolled, 142 had sarcomatoid histology, 68 of these patients were assigned to the atezolizumab/bevacizumab arm, and 74 were assigned to the sunitinib arm. The study noted that patients with sarcomatoid histology have higher expression of PDL1 (with 61\% expressing PDL1) and more likely to have intermediate to poor-risk disease. The data analysis of this subgroup showed a higher ORR in the atezolizumab/bevacizumab arm at $49 \%$ vs. $14 \%$ in the sunitinib arm. The CR was higher in the combination arm $10 \%$ vs. only $3 \%$ in the sunitinib arm. The median PFS for sarcomatoid histology was 8.3 months in the atezolizumab/bevacizumab arm compared to 5.3 months in the sunitinib arm. The HR for PFS was 0.46 (95\% CI $0.28-0.78)$ in the PDL1 positive and $0.56(0.38-0.83)$ in the intention-totreat group. The median OS for sarcomatoid histology was not reported (18.3 months to NA) in the combination arm versus 15 months (8.7 to NA) in sunitinib arm, with HR of $0.56(95 \%$ CI 0.32 - 0.96). The study concluded that patients with sarcomatoid histology treated with atezolizumab/bevacizumab had significantly longer PFS and OS [33].

\section{JAVELIN renal 101}

This is a phase III open-label randomized clinical trial comparing avelumab (10 mg/kg every 2 weeks) plus axitinib (5 $\mathrm{mg}$ orally twice daily) or sunitinib (50 mg orally once daily for 28 days on/14 days off in the treatment of patients with previously untreated patients with advanced RCC. The trial enrolled a total of 886 patients, 442 patients received avelumab plus axitinib, and 444 patients received sunitinib. Of the patients, $63.2 \%$ had PDL1 positive tumors. The ORR was $55.2 \%$ with avelumab plus axitinib and $25.5 \%$ with sunitinib. The subgroup data analysis presented at European Society for Medical Oncology (ESMO) (2019) showed that out of the total enrolled subjects, 108 patients had sarcomatoid RCC, 47 patients received avelumab plus axitinib, and 61 patients received sunitinib. Of these patients, $90 \%$ had undergone prior nephrectomy; $72 \%$ in the combination arm and $85 \%$ in sunitinib arm were PDL1 positive. The ICI/TKI combination arm was reported to have an ORR of $46.8 \%$ compared to $21.3 \%$ in the sunitinib group. A CR was reported among two patients in the combination arm compared to none with sunitinib. Median PFS was prolonged in combination arm with HR of $0.57(95 \%$ CI $0.325-1.003)$ [34].

\section{Conclusions}

sRCC is an aggressive form of RCC that can be associated with any histological subtype of RCC, but most commonly 
seen with clear cell RCC. It is thought to be present in up to $20 \%$ of patients with advanced disease, with the majority of cases having intermediate or poor prognostic factors. Notable features of sRCC are the higher expression of PDL1, and the poor outcomes compared to clear cell RCC when treated with sunitinib. In all the mentioned clinical trials, patients with sRCC had statistically significant higher ORR, PFS, OS and CR when treated with ICIs compared to the previous standard of care monotherapy with sunitinib.

\section{Acknowledgments}

None to declare.

\section{Financial Disclosure}

None to declare.

\section{Conflict of Interest}

None to declare.

\section{Author Contributions}

Ragia Aly: manuscript idea, research, writing. Amandeep Aujla: writing, editing. Sachin Gupta: review, editing. Ruby Gupta: revision, editing. Sorab Gupta: revision, editing. Sheila Khalathil: editing.

\section{Data Availability}

The authors declare that data supporting the findings of this study are available within the article.

\section{References}

1. American Cancer Society: Cancer Facts and Figures 2020. Atlanta, Ga: American Cancer Society, 2020. https://www.cancer.org/research/cancer-facts-statistics/allcancer-facts-figures/cancer-facts-figures-2020.html.

2. Bray F, Ferlay J, Soerjomataram I, Siegel RL, Torre LA, Jemal A. Global cancer statistics 2018: GLOBOCAN estimates of incidence and mortality worldwide for 36 cancers in 185 countries. CA Cancer J Clin. 2018;68(6):394424.

3. Eble JN, Sauter G, Epstein JI, Sesterhenn IA. Pathology and genetics of tumours of the urinary system and male genital organs. Lyon, France: International Agency for Research on Cancer. 2004.

4. Patard JJ, Leray E, Rioux-Leclercq N, Cindolo L, Ficarra V, Zisman A, De La Taille A, et al. Prognostic value of histologic subtypes in renal cell carcinoma: a multicenter experience. J Clin Oncol. 2005;23(12):2763-2771.

5. Moch H, Humphrey PA, Ulbright TM, Reuter VE. WHO classification of tumours of the urinary system.

6. Liang X, Liu Y, Ran P, Tang M, Xu C, Zhu Y. Sarcomatoid renal cell carcinoma: a case report and literature review. BMC Nephrol. 2018;19(1):84.

7. Wei S, Al-Saleem T. The pathology and molecular genetics of sarcomatoid renal cell carcinoma: a mini-review. J Kidney Cancer VHL. 2017;4(2):19-23.

8. Cheville JC, Lohse CM, Zincke H, Weaver AL, Leibovich BC, Frank I, Blute ML. Sarcomatoid renal cell carcinoma: an examination of underlying histologic subtype and an analysis of associations with patient outcome. Am J Surg Pathol. 2004;28(4):435-441.

9. Kyriakopoulos CE, Chittoria N, Choueiri TK, Kroeger N, Lee JL, Srinivas S, Knox JJ, et al. Outcome of patients with metastatic sarcomatoid renal cell carcinoma: results from the International Metastatic Renal Cell Carcinoma Database Consortium. Clin Genitourin Cancer. 2015;13(2):e79-85.

10. Survival outcomes of sarcomatoid renal cell cancer (sRCC). https://ascopubs.org/doi/abs/10.1200/JCO.2020. 38.15_suppl.e17101.

11. Rini $\bar{B}$, Hutson TE, Figlin RA, Lechuga MJ, Valota O, Serfass L, Rosbrook B, et al. Sunitinib in patients with metastatic renal cell carcinoma: clinical outcome according to International Metastatic Renal Cell Carcinoma Database Consortium Risk Group. Clin Genitourin Cancer. 2018;16(4):298-304.

12. Schmidinger M, Porta C, Oudard S, Denechere G, Brault Y, Serfass L, Costa N, et al. Real-world Experience With Sunitinib Treatment in Patients With Metastatic Renal Cell Carcinoma: Clinical Outcome According to Risk Score. Clin Genitourin Cancer. 2020.

13. Savard MF, Wells JC, Graham J, Dudani S, Steinharter JA, McGregor BA, Donskov F, et al. Real-World assessment of clinical outcomes among first-line sunitinib patients with clear cell metastatic renal cell carcinoma (mRCC) by the International mRCC Database Consortium Risk Group. Oncologist. 2020;25(5):422-430.

14. Heng DY, Xie W, Regan MM, Harshman LC, Bjarnason GA, Vaishampayan UN, Mackenzie M, et al. External validation and comparison with other models of the International Metastatic Renal-Cell Carcinoma Database Consortium prognostic model: a population-based study. Lancet Oncol. 2013;14(2):141-148.

15. Mian BM, Bhadkamkar N, Slaton JW, Pisters PW, Daliani D, Swanson DA, Pisters LL. Prognostic factors and survival of patients with sarcomatoid renal cell carcinoma. J Urol. 2002;167(1):65-70.

16. Merrill MM, Wood CG, Tannir NM, Slack RS, Babaian $\mathrm{KN}$, Jonasch E, Pagliaro LC, et al. Clinically nonmetastatic renal cell carcinoma with sarcomatoid dedifferentiation: Natural history and outcomes after surgical resection with curative intent. Urol Oncol. 2015;33(4):166 e121-169.

17. Sharma P, Hu-Lieskovan S, Wargo JA, Ribas A. Primary, adaptive, and acquired resistance to cancer immunotherapy. Cell. 2017;168(4):707-723. 
18. Vesely MD, Kershaw MH, Schreiber RD, Smyth MJ. Natural innate and adaptive immunity to cancer. Annu Rev Immunol. 2011;29:235-271.

19. Chen DS, Mellman I. Oncology meets immunology: the cancer-immunity cycle. Immunity. 2013;39(1):1-10.

20. Freeman GJ, Long AJ, Iwai Y, Bourque K, Chernova T, Nishimura H, Fitz LJ, et al. Engagement of the PD-1 immunoinhibitory receptor by a novel B7 family member leads to negative regulation of lymphocyte activation. J Exp Med. 2000;192(7):1027-1034.

21. Wei SC, Duffy CR, Allison JP. Fundamental mechanisms of immune checkpoint blockade therapy. Cancer Discov. 2018;8(9):1069-1086.

22. Kawakami F, Sircar K, Rodriguez-Canales J, Fellman BM, Urbauer DL, Tamboli P, Tannir NM, et al. Programmed cell death ligand 1 and tumor-infiltrating lymphocyte status in patients with renal cell carcinoma and sarcomatoid dedifferentiation. Cancer. 2017;123(24):4823-4831.

23. Joseph RW, Millis SZ, Carballido EM, Bryant D, Gatalica Z, Reddy S, Bryce AH, et al. PD-1 and PD-L1 expression in renal cell carcinoma with sarcomatoid differentiation. Cancer Immunol Res. 2015;3(12):1303-1307.

24. Shin SJ, Jeon YK, Kim PJ, Cho YM, Koh J, Chung DH, Go H. Clinicopathologic analysis of PD-L1 and PD-L2 expression in renal cell carcinoma: association with oncogenic proteins status. Ann Surg Oncol. 2016;23(2):694-702.

25. Arora E, Masab M, Mittar P, Jindal V, Gupta S, Dourado C. Role of Immune Checkpoint Inhibitors in Advanced or Recurrent Endometrial Cancer. Cureus. 2018;10(4):e2521.

26. Vaddepally RK, Kharel P, Pandey R, Garje R, Chandra AB. Review of indications of FDA-approved immune checkpoint inhibitors per NCCN guidelines with the level of evidence. Cancers (Basel). 2020;12(3):738.

27. Shuch B, Bratslavsky G, Linehan WM, Srinivasan R. Sarcomatoid renal cell carcinoma: a comprehensive review of the biology and current treatment strategies. Oncologist. 2012;17(1):46-54.

28. Motzer RJ, Tannir NM, McDermott DF, Aren Frontera O, Melichar B, Choueiri TK, Plimack ER, et al. Nivolumab plus ipilimumab versus sunitinib in advanced renal-cell carcinoma. N Engl J Med. 2018;378(14):1277-1290.

29. McDermott DF, Choueiri TK, Motzer RJ, et al. CheckMate 214 post-hoc analyses of nivolumab plus ipilimum$\mathrm{ab}$ or sunitinib in IMDC intermediate/poor-risk patients with previously untreated advanced renal cell carcinoma with sarcomatoid features.

30. Rini BI, Plimack ER, Stus V, Gafanov R, Hawkins R, Nosov D, Pouliot F, et al. Pembrolizumab plus axitinib versus sunitinib for advanced renal-cell carcinoma. N Engl J Med. 2019;380(12):1116-1127.

31. Tykodi SS, Donskov F, Lee J-L, et al. First-line pembrolizumab (pembro) monotherapy in advanced clear cell renal cell carcinoma (ccRCC): Updated results for KEYNOTE-427 cohort A. Abstract of a poster presented at the American Society of Clinical Oncology 2019 Annual Meeting. Chicago, Illinois. June 3, 2019.

32. Lee JL, Ziobro M, Gafanov R, et al. First-line pembrolizumab (pembro) monotherapy for advanced non-clear cell renal cell carcinoma (NCC-RCC). Journal of Clinical Oncology. 2019;37:4569-4569.

33. Rini BI, Powles T, Atkins MB, Escudier B, McDermott DF, Suarez C, Bracarda S, et al. Atezolizumab plus bevacizumab versus sunitinib in patients with previously untreated metastatic renal cell carcinoma (IMmotion151): a multicentre, open-label, phase 3, randomised controlled trial. Lancet. 2019;393(10189):2404-2415.

34. Motzer RJ, Penkov K, Haanen J, Rini B, Albiges L, Campbell MT, Venugopal B, et al. Avelumab plus axitinib versus sunitinib for advanced renal-cell carcinoma. N Engl J Med. 2019;380(12):1103-1115. 